\title{
VALORACIÓN BIOGEOGRÁFICA DE LOS PAISAJES FORESTALES DE ARTIKUTZA (NAVARRA), A PARTIR DE LA APLICACIÓN DEL MÉTODO DE VALORACIÓN (LANBIOEVA)
}

\author{
P.J. Lozano Valencia, I. Latasa Zaballos y B. Dorronsoro Loiarte \\ Departamento de Geografía, Prehistoria y Arqueología de la UPV/EHU \\ Calle Tomás y Valiente S/N-Vitoria-Gasteiz (01006) \\ pedrojose.lozano@ehu.es \\ itxaro.latasa@ehu.es \\ bedorron@gmail.com
}

\begin{abstract}
Resumen: El presente artículo se basa en un trabajo de investigación desarrollado desde hace más de 20 años y que persigue consolidar un método de inventariación y valoración biogeográfica de diferentes paisajes vegetales en latitudes medias y altas. Hasta la fecha, se ha ido aplicando a diferentes ecosistemas ubicados dentro de la Península Ibérica. No obstante y, de cara a valorar la robustez del método, en los dos últimos años se ha aplicado a otros ámbitos. El presente artículo se centra, expone y analiza los resultados obtenidos en Artikutza, noroeste de Navarra. La metodología se basa en un inventariado sistemático de diferentes unidades junto a una valoración basada en criterios naturales, territoriales, culturales, de manejo, riesgos, etc. Los resultados, en este caso, otorgan una preponderancia clara a los bosques de ribera, seguidos de los robledales y los hayedos. En un segundo grupo se encontrarían los bosques de repoblación: pinares de pino silvestre, roble rojo americano y el abetal-falso cupresal.
\end{abstract}

Palabras clave: valoración biogeográfica, Artikutza, LIC, inventario biogeográfico y LANBIOEVA.

\begin{abstract}
This paper is based on a research work developed over 20 years that aims to consolidate a method for making biogeographical inventories and evaluations of vegetal landscapes in mid and high latitudes. To date, the method has been applied to different ecosystems within the Iberian Peninsula. However, in order to assess the robustness of the method, it has been
\end{abstract}

Recibido: 12-01-2015. Aceptado: 02-07-2015. 
also applied to other areas. This article exposes and analyzes the overall results obtained in the area of Artikutza, Northwest of Navarre. The methodology is based on a systematic inventory of different units along with an assessment based on natural, territorial, cultural, management and risks criteria. The results, in this case, show a clear preponderance of the riparian forests, followed by oak and beech forests. A second group would be plantation forests: wild pine forest, northern red oak and fir-false cupresal.

Keywords: biogeographical evaluation, Artikutza, Site of Community Interest (SCI), biogeographic inventory \& LANBIOEVA.

\section{Introducción}

Uno de los objetivos básicos de la Geografía y, como disciplina de la misma; la Biogeografía, debe ser generar los conocimientos, herramientas metodológicas y resultados necesarios que aporten a la sociedad, no sólo conocimiento, sino aplicación a los territorios y políticas de ordenación y gestión de los mismos.

En las últimas siglos se asiste a una reducción drástica de determinados ecosistemas y, junto a ellos, uno de los mayores problemas ambientales; la reducción de la diversidad a escala planetaria y la extinción de especies a un ritmo mucho más elevado que el estimado en tiempos pretéritos. Frente a los evidentes peligros que pueden resultarse de un crecimiento económico ilimitado, hace falta generar protocolos de análisis, valoración y diagnóstico ambiental, lo más objetivo posibles y que aporten un necesario cambio de paradigma hacia políticas territoriales, económicas, sociales y ambientales mucho más equilibradas y basadas en la verdadera capacidad de los ecosistemas de generar recursos, por su puesto limitados y que, por lo tanto, se trata de planificar y gestionar adecuadamente (Mc Neill, 2000).

De hecho, los territorios a conservar no sólo presentan valores medioambientales, sino que implican y afectan a una serie de agentes, relaciones, visiones e intereses sociales, económicos, políticos, productivos, etc. para los que hay que procurar herramientas de valoración y gestión con visiones que van más allá de los simples valores naturales a conservar.

Conscientes de esas necesidades, desde hace más de 20 años se ha trabajado, a partir de distintos proyectos de investigación, en la línea de generar una herramienta potente y científicamente robusta que permita inventariar, analizar, diagnosticar, valorar y realizar las propuestas necesarias para la correcta gestión de diferentes paisajes, unidades ambientales o ecosistemas. Dicha propuesta se resume bajo el acrónimo LANBIOEVA (Landscape Biogeographic Evaluation). 
El presente artículo recoge los resultados de inventariar y valorar, durante más de 7 meses y de manera sistemática, una serie de paisajes forestales ubicados dentro del Lugar de Interés Comunitario (en adelante LIC) de Artikutza. Se trata de un espacio muy peculiar por el manejo que durante los últimos 100 años ha disfrutado. El ayuntamiento de Donostia-San Sebastian compró la finca para destinarla a la construcción de un pantano que surtiera y garantizara el suministro de agua de boca de gran calidad para la ciudad. Esto ha conllevado una política de protección de la vegetación a ultranza, de manera que la buena calidad de las masas forestales garantizara una buena calidad hídrica.

El LIC de Artikutza cuenta con 3638 Ha. y se ubica dentro de la Comunidad Autónoma de Navarra (CAN), en concreto en su vértice noroccidental dentro del munici-

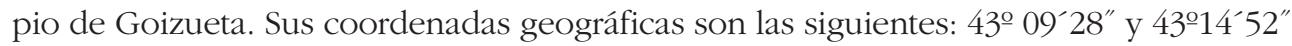
(latitud norte) y 01은 $45^{\prime} 35^{\prime \prime}$ y $1^{\circ}$ 49 $30^{\prime \prime}$ (longitud oeste). Las coordenadas UTM son las siguientes: 4.789 .076 norte, 4.779 .084 sur, 595.338 este, 600.725 oeste (Ekilan, 2012).

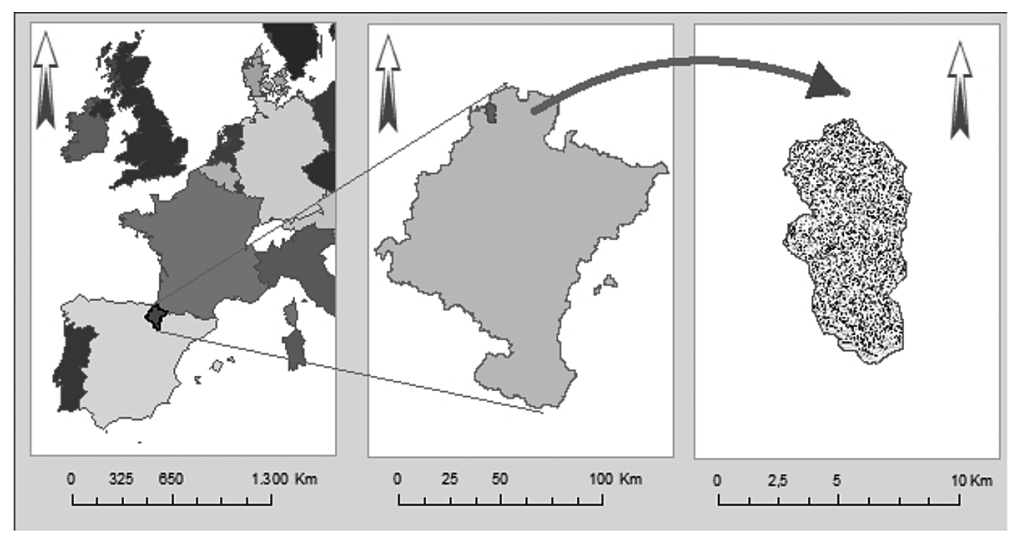

Figura 1. Ubicación geográfica del LIC de Artikutza.

Fuente: Elaboración propia.

La estructura territorial de Artikutza muestra una peculiaridad; se trata de una morfología en forma de un circo montañoso que recorre todo el perímetro de la finca. Dicho perímetro es irregular puesto que las altitudes de los diferentes montes cuentan con cotas muy desiguales. Mientras el punto más elevado viene representado por el monte Arainburu (1053 m), el más bajo aparece junto al talweg del río Artikutza con 255 m. Este es el único sector donde se rompe el mencionado cierre perimétrico montañoso. En total cuenta con una extensión perimetral de 30 kilómetros (Aizpuru et al., 1989).

El LIC de Artikutza cuenta además con características mesológicas ciertamente originales. Para empezar muestra una litología pizarrosa, grauwaquica y calcárea ciertamente antigua. Se trata de litologías sedimentadas durante el ciclo herciniano y, más con- 
cretamente, durante los últimos estadios del Devónico y el Carbonífero, hace 320 millones de años. Los lentejones calcáreos muestran unas calizas tremendamente metamorfizadas y marmorizadas. Las otras dos morfologías son relativamente deleznables aunque las grauwacas muestran una mayor competencia a la erosión con lo que aparecen configurando los relieves más vigorosos. A su vez, la existencia, en las inmediaciones, del batolito granítico más occidental del pirineo axial (Peñas de Aya) da lugar a sectores con litología granítica. Dentro de ellos, dispuestos en el sector más septentrional del LIC, también aparecen cotas elevadas (800-900 m). La red hidrográfica, por su parte, se ha encajado notablemente, muestra una estructura dendrítica muy densa y, en general, el espacio cuenta con altas pendientes. El 90\% del LIC se encuentra entre los 300 y 800 metros y el 90,2\% de la reserva (3.282 ha) cuenta con un porcentaje superior al $35 \%$ de pendiente. El 44,9\% (1.634 ha) muestra una pendiente superior al 55\%.

En lo que respecta a las condiciones climáticas, se puede hablar de un régimen templado, de carácter oceánico donde las temperaturas son relativamente frescas en verano y no excesivamente bajas en invierno. De hecho, la masa oceánica, en este caso el mar cantábrico y, más concretamente, el sector más oriental del Golfo de Vizcaya, cuenta con una influencia notable al situarse a menos de $20 \mathrm{~km}$ de Artikutza. La temperatura media es de $15^{\circ} \mathrm{C}$. En cualquier caso, la temperatura media del mes más frío nunca desciende de los $5^{\circ} \mathrm{C}$, mientras que la del mes más caluroso nunca excede de los $20^{\circ} \mathrm{C}$. Pero si la masa oceánica ejerce una influencia fundamental en las temperaturas, no la ejerce en menor medida sobre las precipitaciones. Artikutza se configura como el punto de la Península Ibérica donde mayores precipitaciones se dan (Lozano, 2006). Esto se debe a tres factores fundamentales; por una parte, la Cordillera Cantábrica hace que los flujos del oeste y noroeste (los dominantes en esta región) choquen contra ella y discurran en paralelo por la costa. Al llegar al sector más occidental del Golfo de Vizcaya chocan contra las estribaciones pirenaicas y se ven obligadas a pasar por estos montes de altitudes mucho más modestas. Por otra parte, estas elevaciones ejercen un papel fundamental al configurarse como una verdadera pantalla que genera abundantes precipitaciones orográficas. Por último, el que la región occidental del Mar Mediterráneo se configure como ciclogeneradora durante gran parte del año, hace que exista un efecto succionador hacia el Valle del Ebro, de manera que, una vez más, las abundantes masas cargadas de humedad dejan las precipitaciones en estos montes llegando al mencionado valle bastante desecadas y generando el famoso cierzo. Con todo, las precipitaciones medias son superiores a los $2.500 \mathrm{~mm}$. anuales no siendo excepcionales los años que sobrepasan los 3000 mm Así, se puede hablar de un régimen templado hiperhúmedo sin estación seca según la clasificación de Koopen (Ekilan, 2012).

Las grandes pendientes junto a las importantes precipitaciones y a los sustratos mayoritariamente ácidos dan lugar a suelos muy escasos y tremendamente lavados, por tanto, oligótrofos y, en general, bastante escasos y pobres. Las clases de suelos más abundantes son los ranker sobre sustrato granítico y los cambisoles distritos sobre las pizarras, grauwacas e incluso las calizas marmóreas. 


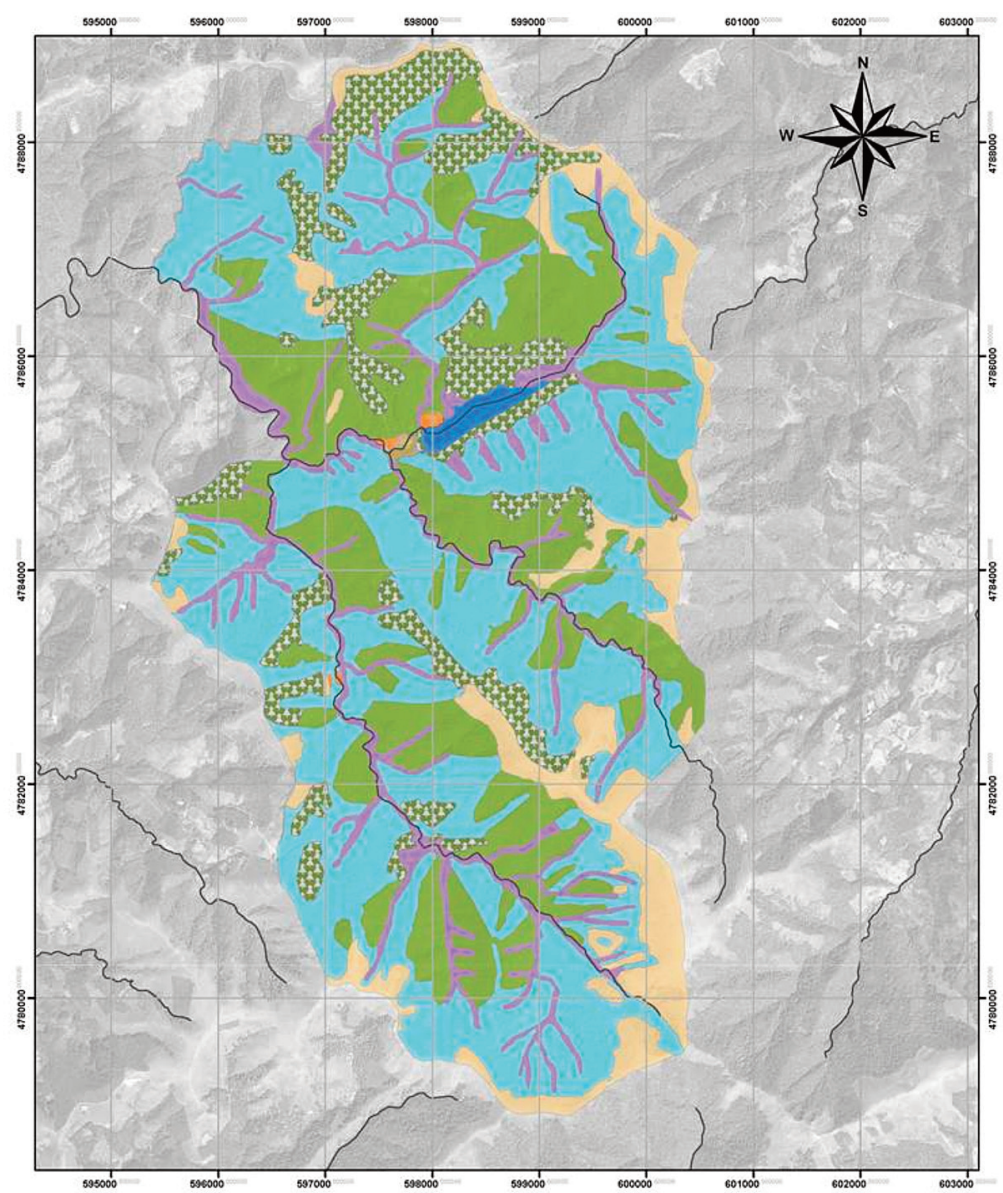

Unidades de vegetación de Artikutza

\section{Leyenda}

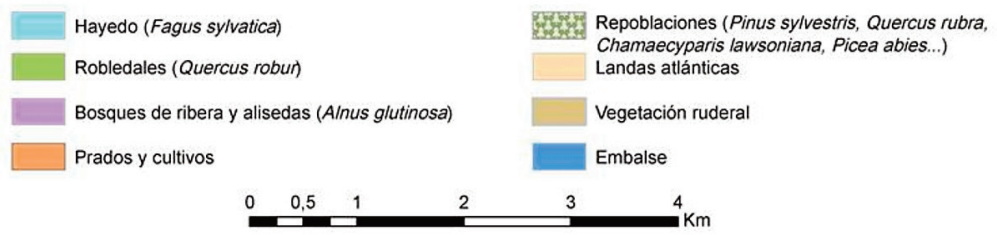

Figura 2. Mapa de vegetación de Artikutza.

Fuente: Elaboración propia a partir de Ekilan, 2012. 
Todas estas condiciones dan lugar a un cortejo de vegetación dividido en pisos y sectores pero que, en general, muestra una cierta pobreza en especies. En realidad más del 90\% del territorio se encuentra cubierto por bosques de manera que casi toda la flora cuenta con un carácter forestal. Por otra parte, las unidades cuentan con una gradación clara en altura. En los talweg y aledaños se sitúan los bosques de ribera donde la especie dominante es el aliso (Alnus glutinosa), mientas en las laderas bajas y medias lo hacen los robledales de Quercus robur. En las laderas medias a altas se da una gradación entre la anterior formación y el hayedo acidófilo de Fagus sylvatica. Por último, la vocación forestal del territorio hizo que antes de la compra por parte del ayuntamiento de Donostia-San Sebastian, una gran parte del mismo estuviera dedicada a repoblaciones silvícolas con dos especies dominantes, por una parte los pinares de Pinus sylvestris y, por otra, los robledales de Quercus rubra. En sectores más concretos también se dieron repoblaciones de abeto rojo Picea abies y falso ciprés Chamaecyparis lawsoniana. De forma más dispersa y con muy poca representación también se pueden encontrar rodales de Robinia pseudoacacia y Larix sp.

\section{Estado de la cuestión}

La presente aportación se enmarca en un línea de investigación iniciada hace más de 20 años, encaminada al diseño y puesta a punto de propuestas metodológicas de inventariación y valoración biogeográfica de comunidades y paisajes bióticos, que se ha ensayado en diferentes ámbitos territoriales europeos (Península Ibérica, Balcanes, Península Escandinava...) y centro-sudamericanos (Nicaragua, Región Mediterránea de Chile, Patagonia...) (Cadiñanos y Meaza, 1998b; Cadiñanos y Meaza, 2000; Cadiñanos, Meaza y Lozano, 2002; Cadiñanos et al., 2002; Meaza, Cadiñanos y Lozano, 2006; Lozano et al., 2007; Lozano y Cadiñanos, 2009; Cadiñanos, Lozano y Quintanilla, 2011; Lozano et al., 2013; Lozano et al., 2015).

Es interesante reseñar que la presente propuesta metodológica, de raigambre geográfica, constituye un modelo de aproximación al ejercicio valorativo concurrente y complementario con el de otras líneas de trabajo de enfoques muy diversos. Es el caso de trabajos como el de Constanza et al. (1997), que realizan una aproximación a la valoración de los servicios ambientales que ofrecen distintos ecosistemas desde la perspectiva de diferentes profesionales, no exenta de discusión puesto que valorar monetariamente algunos servicios naturales enfrenta poderosamente a la comunidad científica y técnica. Lo es, asimismo, el de la valoración de los ecosistemas y paisajes a través de los estudios cuantitativos relacionados exclusivamente con la biodiversidad (Wittaker, 1972) medida bajo tres parámetros -cantidad de especies, población de cada una de ellas y diversidad génica (Benton, 2001) - y que obvia criterios de índole cultural frecuentemente tan importantes como los de orden natural en ecosistemas y paisajes profundamente manejados y modificados por la acción humana. 
La valoración de los ecosistemas como activo capital está bien estudiada desde una perspectiva natural o social (como productor de bienes y alimentos; soporte vital; bien espiritual; e incluso como reservorio genético para un uso futuro no determinado en el momento presente). No obstante, otras formas de capitalización de este recurso no reconocen estos valores y consecuentemente la valoración de los servicios de los ecosistemas no es tenida en cuenta por el gran capital y la economía de mercado (Daily, G.C. et al., 2000). En este mismo trabajo los autores elaboraron una interesante propuesta de valoración ambiental basada en cuantificar los servicios de los ecosistemas, analizando que el precio de mercado nunca refleja el coste de producción social y que la mayoría de los servicios ecosistémicos jamás se incorporan a la valoración del coste de conservación de un ecosistema. Por tanto, en términos estrictamente económicos, la conservación de un ecosistema siempre ofrece un resultado de coste y no de beneficios pecuniarios.

Fisher et al. (2009) parten de que los servicios de los ecosistemas no son homogéneos en los diferentes paisajes. Esta heterogeneidad es posteriormente desarrollada por De Groot et al (2002), aunque no profundiza en la descripción de cómo las relaciones espaciales pueden ser tenidas en cuenta en el proceso de valoración (Blaschke, 2005). En este sentido, Syrbe y Walz (2012) plantean que los indicadores para la evaluación de los servicios ecosistémicos deberían estar irremediablemente relacionados con su recurrente implicación espacial. En tal sentido, se equipara, en términos de valoración, los servicios ecosistémicos a los servicios de paisaje, considerándolos sinónimos (Syrbe y Walz, 2012). No obstante, solo se emplea la terminología paisajística si la apreciación de relaciones espaciales es evidente y constatable.

Frecuentemente, estos estudios se han centrado en ejercicios científicos relativamente complejos y difíciles de interpretar y utilizar por el gestor que, a la vista de las características físicas, ambientales, mesológicas, culturales, patrimoniales, percepcionales, etc., debe poner en marcha los preceptivos planes para la ordenación y gestión de los paisajes y territorios, fundamentalmente los de dominante natural. De ahí que sean altamente interesantes las aproximaciones metodológicas que ofrezcan al mencionado gestor una herramienta operativa para la toma responsable de decisiones (Strijker, Sijtsma y Wiersma, 2000; Debinski, Ray y Saveraid, 2001) y, sobre todo, las que ofrezcan una visión transversal que combine cuestiones relacionadas con los valores naturales intrínsecos de las unidades de paisaje con aquellas otras ligadas a los procesos ecológicos y con las que atañen a los aspectos culturales y de manejo del territorio.

Según Cáncer Pomar (1999), la valoración del paisaje puede simplificarse en dos vertientes completamente diferenciadas: la valoración científica y la valoración social. La primera ha de ser elaborada por especialistas de diferentes ramas de la ciencia, que en opinión de Muñoz Jiménez (1981), deben filtrar la información para que su percepción no sea distorsionada, por consiguiente, el control de los filtros es lo que permite diferenciar la percepción científica de la percepción vulgar. No obstante, esta corriente de investigación que se inició en EEUU en los años 60, puso todo su énfasis en la consi- 
deración de las cualidades ecológicas del territorio (aproximación a una situación climácica) y en el mantenimiento de sus rasgos de naturalidad (Cáncer Pomar, 1999). Dentro de esta corriente y como respuesta al método calificado como ecológico (McHarg, 1969; Leopold, 1969) surge el método de estética formal (Litton, 1972; Wright, 1974) donde los arquitectos paisajistas introducen la valoración de las cualidades estéticas, sobre todo en espacios con alto grado de antropización.

En cuanto a la valoración social del paisaje, cabe indicar que los trabajos sobre percepción social del paisaje son bien conocidos desde sus inicios en la década de los años 60 , aunque adquirieron una relativa importancia durante las décadas siguientes con los trabajos de Zube et al. (1975), Kaplan y Kaplan (1989) o Bourassa (1990). Estos trabajos consistieron básicamente en valorar las preferencias paisajísticas de personas pertenecientes a diferentes culturas. La práctica totalidad de estos trabajos ofrecieron unos resultados similares, concluyendo que los paisajes naturales gozaban de una mejor valoración en términos de percepción que los paisajes en los que se reconocía alguna influencia humana (Ulrich, 1993).

Otras metodologías de evaluación del territorio, caso de la Evaluación Ambiental Estratégica (De la Barrera et al., 2011) pueden ser perfectamente aplicables a la toma de decisiones al tener en consideración el alcance del impacto que se puede ocasionar al territorio en base a la aplicación de ciertas políticas (Oñate et al., 2002; Hervé, 2010). En trabajos recientes desarrollados en Chile, como el de De la Barrera et al. (2011) se parte de una evaluación ecológica tomando como elementos evaluables los definidos como fragmentos (patches) o hábitats definidos como contenedores de biodiversidad y, consecuentemente como contenedores de ecosistemas (Forman, 1995; Grez et al., 2006; Pauchard et al., 2006).

Estos tres últimos trabajos muestran una metodología muy enfocada desde la Ecología del paisaje hacia una evaluación de la biodiversidad en tres escalas principales: genética, taxonómica y espacial, siendo esta última representada por los ecosistemas y/o los paisajes y donde los elementos evaluables son su composición, estructura y función (Noss, 1990; Serey et al., 2007). No obstante, esta propuesta sostiene que los estudios de vegetación en términos de madurez ecológica, grado de intervención antrópica y capacidad de acogida para la fauna (Ausden, 2007; Hagar, 2007; Adams et al. 2009; Drever y Martin, 2010) son útiles para la gestión ambiental del territorio, pero a su vez complementarios de la propuesta de análisis de la biodiversidad a través del paisaje.

Por el contrario, en nuestra propuesta, el análisis del paisaje vegetal es la base sobre la que se procede a la valoración del paisaje. Por consiguiente, el modelo de inventario es más exhaustivo y proceloso que los fitosociológicos o similares; pero, una vez realizado, la información obtenida es notablemente superior, lo que permite una caracterización geobotánica y biogeográfica mucho más fiable y, en última instancia, un diagnóstico más fino de la calidad del medio con fines fundamentalmente conservacionistas. 
Por su parte, el modelo de valoración trata de ofrecer una metodología coherente, rigurosa, versátil y práctica, basada en pautas sencillas, flexibles y claras, con resultados estándar fáciles de aplicar e interpretar de cara a una correcta y jerárquica gestión de las comunidades y paisajes bióticos concernidos. Desde esta perspectiva, es un importante instrumento en la ordenación y gestión territorial, una herramienta fundamental para el conocimiento y la toma de decisiones respecto a los paisajes vegetales considerados como patrimonio natural y cultural.

\section{Objetivos}

El propósito del presente trabajo es presentar los resultados de la aplicación del mencionado método a la evaluación de los paisajes forestales de Artikutza. Su contenido y funcionalidad práctica llevan aparejados los siguientes objetivos operativos:

- La integración de una visión que considere los múltiples atributos ambientales que conforman los paisajes forestales de Artikutza a inventariar y valorar.

- La generación de protocolos de valoración biogeográfica que recojan diferentes aspectos como: geología, geomorfología, suelos, vegetación, fauna, usos del suelo, etc. dentro del territorio de estudio que nos ocupa.

- Valoraciones parciales que puedan ser tenidas en cuenta de forma sectorial atendiendo a los atributos o cuestiones que se consideren oportunas a la hora de planificar y gestionar el LIC de Artikutza: valores naturales, culturales, mesológicos, amenazas, etc.

- La creación de un modelo metodológico de inventariación y valoración, lo más sencillo posible, para que pueda ser desarrollado y aplicado en otros espacios.

- La experimentación del marco metodológico ya aplicado a ámbitos como la Península Ibérica, la zona Mediterránea de Chile, la Patagonia, la Península Balcánica, etc. en este caso en el LIC de Artikutza.

\section{Metodología utilizada}

Una de las principales vertientes de la Biogeografía Aplicada es, sin duda, la valorativa, que trata de constatar el estado actual de la vegetación para su evaluación cualitativa con fines, principalmente, conservacionistas. Desde esta perspectiva, es un importante instrumento en la Ordenación y Gestión Territorial, una herramienta fundamental para el conocimiento y la toma de decisiones respecto a la vegetación considerada como patrimonio natural y cultural. El análisis valorativo de la vegetación constituye, por otra 
parte, el punto de encuentro de especialistas provenientes de distintos campos del conocimiento -botánicos, ecólogos, geógrafos, ingenieros y otros técnicos y profesionales (Cadiñanos \& Meaza, 1998a y 1998b)-, lo que hace deseable llegar a la elaboración de procedimientos consensuados y asumibles por la generalidad de los expertos.

\subsection{Inventariado}

Una vez predefinidas las unidades y su ubicación, a través de un laborioso trabajo de fotointerpretación y campo, se estimó un número mínimo de inventarios diseminados con carácter estratificado y aleatorio por el SIG (ARCGIS.10). A su vez, el número de inventarios a realizar para cada una de las unidades, de cara a que de todos ellos se genere el sininventario tipo de esa unidad concreta, depende de los siguientes criterios:

a) La superficie que cada paisaje forestal ocupa dentro del LIC de Artikutza. Cuanto mayor es ésta mayor cantidad de inventarios le corresponden.

b) Se tiene en cuenta, también, la cantidad de facies diferentes que puede presentar la unidad en cuestión. Cuanto mayor número de variantes mayor será el número de inventarios. A modo de ejemplo, en este caso los bosques de ribera han sido la unidad que mayor variedad registra, de manera que aunque en superficie ocupan poco, se ha hecho un número mayor de inventarios, en proporción.

c) El número de inventarios se ha determinado, también, atendiendo al número de especies nuevas que aparecían entre los inventarios correlativos. De esta manera, si entre un inventario y los dos siguientes no existía un número superior al $5 \%$ de plantas nuevas, se estimaba que ese número de inventarios era suficiente. Por poner un ejemplo, en el caso de los abetales-cupresales en los tres inventarios realizados no existía más que un $2 \%$ de plantas nuevas con lo que ese ha sido el número de parcelas seleccionadas para la obtención del consiguiente sininventario.

d) En el caso de los paisajes azonales como el del bosque de ribera también se ha atendido a la obtención de, al menos, un inventario por cada una de las cuencas fluviales más importantes del territorio.

De esta manera, se han caracterizado y evaluado un total de 35 inventarios inéditos realizados entre mayo y noviembre de 2014. En primer lugar y, para cada uno de los inventarios realizados, se obtienen los datos de localización e identificación del lugar (coordenadas UTM, topónimos, etc.), aspectos y rasgos geográficos y medioambientales generales (topográficos, litológicos, geomorfológicos, edáficos e hidrológicos), fotografías de la parcela, etc. A continuación se tomaban no sólo los habituales datos sobre todos los taxones de la flora vascular presentes, sino también de la flora fúngica y liquénica (hongos y líquenes) y la cobertura y algunas especies de la briófita (estrato muscinal), con indicación de la cobertura de cada especie con una escala de 6 clases (5: máximo, +: mínimo), por cada uno de los cuatro estratos en que dividimos con- 
vencionalmente las comunidades (estrato $>5 \mathrm{~m}$, estrato entre 4,9 y $1 \mathrm{~m}$, estrato entre 0,9 y $0,5 \mathrm{~m}$ y estrato inferior $<0,5 \mathrm{~m}$ ) y la cobertura global. También se han anotado los datos necesarios para la localización e identificación del lugar de registro, las características geográficas (topográficas, litológicas, geomorfológicas, edáficas, hidrológicas, etc.), se han tomado las coordenadas, muestras y fotografías pertinentes, etc.

Además se han tomado una serie de datos imprescindibles para la valoración complementaria de las comunidades forestales. Así, la cobertura global y la riqueza por estratos (COBEST y RIQUEST), la diversidad de hábitats no desglosables (FORHAB), la superficie de la mancha homogénea (FORESP), la variedad dasonómica tipológica (FORFIS, que siempre es 0 en las comunidades de herbáceas), y los valores culturales y etnográficos añadidos (FORCUL).

Con toda la información obtenida por cada uno de los inventarios se confeccionaba el sininventario que, como se ha dicho anteriormente, responde a la caracterización media de la unidad estudiada. Los datos de cobertura, además, se obtienen a través de las medias de los datos registrados por cada especie en cada uno de los inventarios. Esta cuestión añade un mayor grado de objetividad a la formación final y, por tanto, al propio sininventario.

Como es fácil advertir, estos inventarios requieren más tiempo que los fitosociológicos o similares; ahora bien, una vez realizados, la información obtenida es mucho mayor, lo que permite su posterior utilización no sólo para la caracterización geobotánica de la zona en cuestión, sino también para reflejar su disposición estructural y biogeográfica, así como, en última instancia, para realizar la evaluación y gestión de la vegetación, fauna y paisaje.

\subsection{Valoración biogeográfica}

En la figura 3 se pueden observar los diferentes criterios de valoración de forma gráfica.

El objetivo de este método es presentar, desde esa perspectiva integradora, una metodología valorativa coherente, rigurosa y práctica, basada en pautas sencillas, flexibles y claras. Supone, además, la última versión de un esfuerzo sostenido de años (Cadiñanos \& Meaza, 1998a y 1998b; Cadiñanos, Meaza \& Lozano, 2002; Cadiñanos et al., 2002; Cadiñanos \& Lozano, 2006, Lozano et al, 2007; Cadiñanos, Lozano \& Quintanilla, 2011, Lozano et al, 2015) dirigido a perfeccionar procedimientos de valoración fiables y versátiles que tengan validez para el mayor número de ambientes posible y que puedan ser utilizados, indistintamente, por estudiosos de la ciencia de la vegetación, por expertos y profesionales y por las administraciones. La propuesta metodológica descansa en dos conceptos valorativos diferenciados que constituyen, al tiempo, eslabones estrechamente ligados del sistema operativo: 


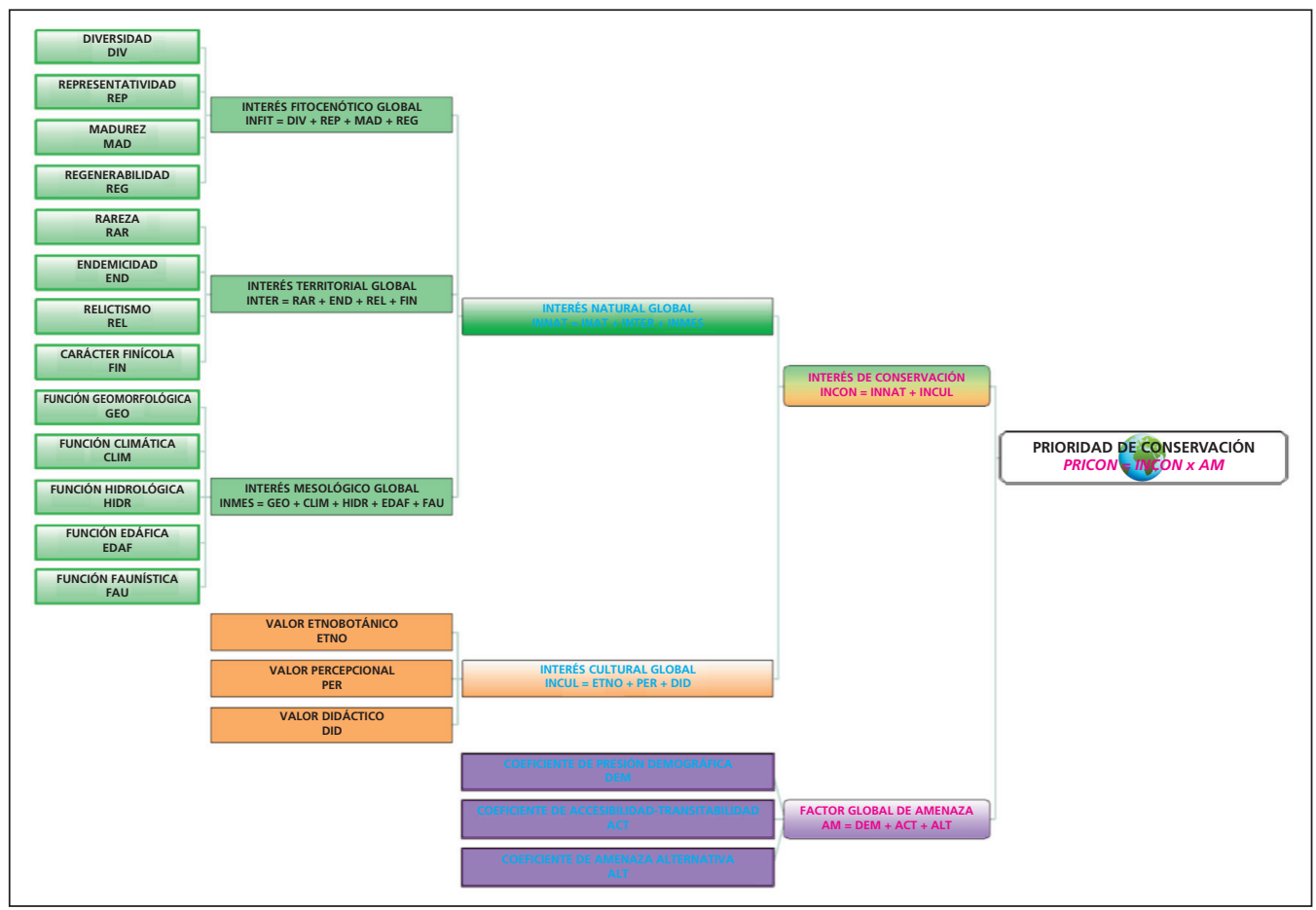

Figura 3. Esquema del método de valoración biogeográfica.

Fuente: Elaboración propia a partir de Ekilan, 2012.

4.2.1. El Interes Natural Global (INNAT): Compuesto por tres tipos de criterios que, posteriormente se desarrollaran pero que abarcan el interés fitocenótico, el interés territorial global y el interés mesológico global.

4.2.1.1. Interes Fitocenótico Global (INFIT): Los criterios fitocenóticos estiman caracteres intrínsecos de la vegetación y del paisaje tales como la diversidad, representatividad, madurez y regenerabilidad espontánea. Como consecuencia, la unidad valorada puede obtener un INFIT que puede variar entre 5 y 50 puntos siguiendo la siguiente fórmula:

$$
\text { INFIT }=\text { DIV (1 a 10) + REP (1 a 10) + MAD (2 a 20) + REG (1 a 10) }
$$

4.2.1.2. Interés Territorial Global (INTER): Los criterios territoriales son bifactoriales -se aplican tanto a nivel de especie como de agrupación- y consideran los atributos de rareza, endemismo, relictismo y carácter finícola, tanto de los taxones presentes como de la propia formación o unidad de paisaje. Consecuencia de ello, la unidad 
valorada puede obtener un INTER que puede variar entre 0 y 50 puntos siguiendo la siguiente fórmula:

INTER $=$ RAR $(0$ a 20) + END $(0$ a 10) + REL (0 a 10) + FIN (0 a 10)

4.2.1.3. Interés Mesológico Global (INMES): Los criterios mesológicos evalúan la contribución de la vegetación a la protección, equilibrio y estabilidad de la biocenosis, el hábitat y el geo-biotopo en el que radica. En su virtud, se proponen 5 parámetros, correspondientes a las funciones geomorfológica, climática, hidrológica, edáfica y faunística (Cadiñanos y Meaza, 1998). Consecuencia de ello, la unidad valorada obtiene un INMES que puede variar entre 6 y 60 puntos siguiendo la siguiente fórmula:

INMES= GEO (2 a 20)+CLIM (1 a 10)+HIDR (1 a 10)+EDAF (1 a 10)+FAU (1 a 10)

La suma de estos tres criterios da lugar al denominado Interés Natural Global (INNAT) y, por tanto, puede ser utilizado como un criterio de raigambre y naturaleza puramente ambiental. En cualquier caso, dicho parámetro puede oscilar entre 11 y 110 puntos. Su fórmula sería:

$$
\text { INNAT }=\text { INFIT }(5 \text { a 50) + INTER (0 a 50) + INMES (6 a 60) }
$$

4.2.2. El Interes Natural Forestal (ITNATFOR): A los valores naturales se le suman parámetros relacionados con la cobertura de los diferentes estratos del bosque (COBEST), la diversidad específica dentro de cada estrato (RIQEST), la superficie continua de la unidad estudiada (FORESP) o la diversidad de microhábitats (FORHAB).

4.2.3. Valoración del Interés Cultural (INCUL): Los criterios de carácter cultural han sido obviados o infrautilizados en la mayor parte de las propuestas valorativas debido, básicamente, al reduccionismo naturalístico. Sin embargo, concitan una atención cada día mayor en la sensibilidad y políticas conservacionistas. El INCUL se calcula teniendo en cuenta, a su vez, otros tres valores diferentes.

4.2.3.1. Valor Etnobotánico (FORETNO): Este criterio trata de evaluar los aspectos etnoculturales (históricos, arqueológicos, religiosos, mitológicos, simbólicos, recreativos, medicinales...) de las plantas, la vegetación y el paisaje que, en su caso, pueden contribuir a hacerlas acreedoras de conservación: vestigios, estructuras y microtopografías relictuales de prácticas forestales (morfología de fustes y ramaje; muros, lezones, setos, caballones y cárcavas de contención o de separación de parcelas; carboneras...), agroganaderas o preindustriales (ferrerías, molinos, aceñas, batanes...) configuradoras de paisajes vegetales peculiares. Se recomienda adjudicar 1 punto por cada elemento considerado de alto valor etnobotánico, respetando siempre la escala de 1 a 10 puntos. El FORETNO es multiplicado por un factor de corrección de 2 puesto que cuenta con mayor importancia que los otros tres, de manera que puede fluctuar entre 0 y 20 (Cadiñanos y Meaza, 1998a). 
4.2.3.2. Valor Percepcional (PER): Es un parámetro que trata de valorar la relación perceptiva (escénica, estética, incluso vivencial) del hombre con respecto a la vegetación. Para su correcta evaluación, lo ideal es contar con encuestas objetivas de preferencias, gustos, querencias y afinidades. El PER fluctúa entre 1 y 10 puntos (Cadiñanos y Meaza, 1998a).

4.2.3.3. Valor Didáctico (DID): Este criterio trata de aquilatar el interés pedagógico del paisaje en sus aspectos naturales y culturales y en la educación y concienciación ambiental de la población en general. Se propone la utilización de la siguiente escala genérica que el investigador habrá de aplicar usando pautas previamente establecidas: desde el valor 1 a aquellas unidades que cuenten con un valor didáctico muy bajo hasta el 10 a aquellas que obtengan uno de muy alta estima. De esta forma el DID puede fluctuar entre 1 y 10 (Cadiñanos y Meaza, 1998a).

Con todo, el Interés Cultural (INCUL) deriva de la suma de las calificaciones adjudicadas a los 3 criterios valorativos que lo integran. Esto es:

INCUL $=$ ETNO $(2$ a 20$)+\operatorname{PER}(1$ a 10$)+$ DID $(1$ a 10$)$

El interés cultural global oscila, entonces, entre 4 y 40 puntos.

4.2.4 El interés de conservación de una determinada agrupación vegetal o paisaje (INCONTFOR) resulta de sumar a la puntuación de INNATFOR (11 a 160) la calificación obtenida por INCUL ( 4 a 40), con lo que el rango de INCON oscila entre 15 y 200 puntos.

4.2.5 La Prioridad de conservación (PRICON). Como se puede apreciar en los siguientes párrafos, es solidaria pero, al tiempo, sustancialmente diferente a la de INCON (interés de conservación), ya que incluye consideraciones ajenas, extrínsecas, a este último. Su resultado ha de ser asumido de manera independiente y no debe ser confundido con él. La prioridad de conservación está, pues, expresamente ideada para su utilización por la administración competente o el gestor, quienes precisan de un diagnóstico claro y operativo sobre cuáles son los espacios que deben ser priorizados cara a su protección y cuáles pueden esperar.

El grado de amenaza que pesa sobre las unidades de vegetación o paisajes concernidos en el proceso evaluativo se calibra en función de tres parámetros: presión demográfica, accesibilidad-transitabilidad y amenaza alternativa.

4.2.5.1. El Coeficiente de Presión Demográfica (DEM): introduce la variable demográfica humana en el sistema valorativo. En su virtud, se priman o penalizan situaciones de alta o baja densidad de población, con mayor o menor peligro, respectivamente, de alteración de la vegetación. La escala a aplicar se obtiene en función de los rangos de densidad real en habitantes/ $\mathrm{km}$ de la zona de estudio. El investigador debe tener 
en cuenta cuestiones como la demografía de la zona, cercanía a grandes núcleos de población y conurbaciones y flujos estacionales, así como la disponibilidad y nivel de detalle de las fuentes estadísticas. La escala propuesta varía entre el 1 para aquellos ámbitos con densidades de menos de 50 habitantes por $\mathrm{km}$ hasta 10 en aquellos que se superen los $450 \mathrm{hab} . / \mathrm{km}^{2}$.

4.2.5.2. El Coeficiente de Accesibilidad-Transitabilidad (ACT) es un parámetro de atención inexcusable a la hora de establecer el nivel de amenaza al que se encuentra expuesta la unidad de paisaje, puesto que la presencia e impronta del ser humano está condicionada por la topografía del terreno, la densidad, tamaño, estado de conservación y grado de penetración de la red viaria y por la estructura más o menos abierta de la unidad valorada; en su caso, también por las limitaciones impuestas por los propietarios o administradores del terreno o por normativa legal dictada por la Administración. La escala propuesta es la que muestra una matriz de doble entrada ( 6 valores de accesibilidad y otros 6 de transitabilidad desde muy baja hasta absoluta para las dos). La combinación de las dos variables va generando puntuaciones que varían desde el 1 hasta 10 cuando la accesibilidad y transitabilidad son absolutas.

4.2.5.3. Coeficiente de Amenaza Alternativa (ALT): se incluyen y calibran bajo este concepto factores alternativos de amenaza que, eventualmente, puedan afectar a la unidad de vegetación o el paisaje objeto de evaluación de manera grave, real y coetánea al ejercicio valorativo -o a muy corto plazo-: catástrofes naturales o provocadas (inundaciones, fuegos), daños palpables por lluvia ácida, vertidos tóxicos o contaminantes, eutrofización, plagas u otras causas de mortalidad excesiva, invasión o desplazamiento de la vegetación original por plantas xenófitas agresivas, desaparición de la vegetación a corto plazo por talas masivas, acondicionamiento para infraestructuras, construcciones, tendidos eléctricos, depósitos, dragados, actividades extractivas, etc. (Olcina, 2004). La escala propuesta varía desde el 1 para la amenaza alternativa muy baja hasta el 10 para aquella que es muy alta.

Así, una vez obtenidos los tres coeficientes se obtiene el Factor Global de Amenaza (AM) sumando los valores de los coeficientes demográfico (DEM=1-10), de accesibilidad-transitabilidad (ACT=1-10) y de amenaza alternativa (ALT=1-10), con lo que el resultado de AM oscila entre 3 y 30 puntos.

La prioridad de conservación (PRICON) de una determinada agrupación vegetal o paisaje se determina multiplicando su valor de INCON (88 a 200) por el coeficiente AM (3 a 30) que le corresponda, con lo que el rango de PRICON oscila entre 264 y 6000 puntos. 


\section{Resultados y discusión}

A partir de los inventarios realizados y, tal y como quedó patente en el capítulo anterior, se dio lugar al sininventario característico para cada paisaje vegetal estudiado. Para cada inventario, a su vez, se genero una valoración biogeográfica, de manera que las que aquí se van a exponer son las medias de cada uno de los parámetros valorados para los diferentes inventarios de cada unidad. Se puede afirmar, por tanto, que responden a la valoración del sininventario de cada paisaje vegetal. No vamos a detenernos en la composición y estructura de las unidades estudiadas puesto que no es objeto de este estudio y además puede consultase en Dorronsoro, Lozano y Latasa (2014). En ese mismo trabajo los paisajes estudiados se dividían en dos grandes grupos. Por una parte aquellos que mostraban la especie dominante a partir de un taxón no introducido y, en el otro grupo, aquellos bosques de repoblación donde dominaba una especie o taxón de carácter alóctono. En cualquier caso, al final estos han sido los paisajes forestales estudiados y el número de inventarios realizados para la obtención del sininventario. Dentro del primer grupo:

- Bosque de ribera: 7 inventarios.

- Robledales (Quercus robur): 6 inventarios.

- Hayedos (Fagus sylvatica): 8 inventarios.

Y dentro del segundo:

- Pinares (Pinus sylvestris): 6 inventarios.

- Roble rojo americano (Quercus rubra): 5 inventarios.

- Abetal-falso cupresal (Picea abies) y (Chamaecyparis lawsoniana): 3 inventarios.

A partir de la obtención de los sininventarios se dan las valoraciones medias. En la tabla 2 aparecen recogidos cada uno de los parámetros valorados y su valor medio. En gris aparecen las diferentes sumas. En primer lugar las sumas parciales de los criterios fitocenológicos, territoriales y mesológicos, que dan lugar a valor natural total (INNAT). En un gris un poco más oscuro se añade la valoración complementaria de las masas forestales, dando lugar al INNATFOR global. A continuación se valoran los distintos parámetros o criterios culturales (con un gris todavía más oscuro) y se suman a los anteriores, de manera que obtenemos el INCONTFOR global (tiene el mismo tono gris que el anterior) y que no deja de ser una suma entre lo natural y lo cultural.

A continuación y, una vez obtenido el valor del interés de conservación (INCONTFOR), se valoran los criterios relacionados con el factor global de amenaza. Éste aparece con el gris más oscuro. EL PRICON o prioridad de conservación se obtendrá a partir de la multiplicación de los dos anteriores dando los valores finales (en negrita). 
Tabla 1. Valoraciones de los distintos parámetros por cada uno de los paisajes forestales de Artikutza

\begin{tabular}{|c|c|c|c|c|c|c|c|c|c|c|c|}
\hline \multirow{2}{*}{\multicolumn{5}{|c|}{$\begin{array}{c}\text { VALORACIÓN } \\
\text { BIOGEOGRÁFICA }\end{array}$}} & & \multirow{3}{*}{$\begin{array}{c}\text { SINBR } \\
\text { PUNT. } \\
6,9\end{array}$} & \multirow{2}{*}{$\begin{array}{l}\text { SINR } \\
\text { PUNT. }\end{array}$} & \multirow{2}{*}{$\begin{array}{l}\text { SINH } \\
\text { PUNT. } \\
\end{array}$} & \multirow{3}{*}{$\begin{array}{c}\text { SINPI } \\
\text { PUNT. } \\
5,5\end{array}$} & \multirow{3}{*}{$\begin{array}{c}\frac{\text { SINRA }}{\text { PUNT. }} \\
4,6\end{array}$} & \multirow{3}{*}{$\frac{\text { SINAB }}{\text { PUNT. }}$} \\
\hline & & & & & PARAMETROS & & & & & & \\
\hline \multirow{33}{*}{ 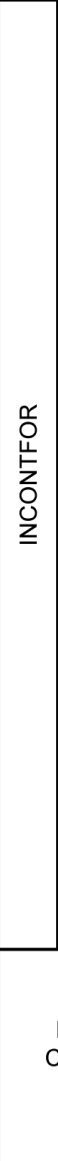 } & \multirow{21}{*}{ 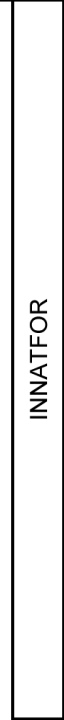 } & \multirow{16}{*}{$\begin{array}{l}\mathfrak{s} \\
\underline{z}\end{array}$} & \multirow{4}{*}{\multicolumn{2}{|c|}{$\frac{\mathfrak{L}}{\underline{\underline{u}}}$}} & DIVERSIDAD & & 5,4 & 4,3 & & & \\
\hline & & & & & MADUREZ (x2) & 18,0 & 17,7 & 18,0 & 14,0 & 12,4 & 10,0 \\
\hline & & & & & REGENERABILIDAD & 7,4 & 7,0 & 7,0 & 2,5 & 3,4 & 1,0 \\
\hline & & & & & SUMA (INFIT GLOBAL) & 32,3 & 30,1 & 29,3 & 22,0 & 20,4 & 15,0 \\
\hline & & & \multirow{5}{*}{\multicolumn{2}{|c|}{$\begin{array}{l}\stackrel{\underline{w}}{\rightleftarrows} \\
\underline{\mathbf{z}}\end{array}$}} & RAREZA & 1,9 & 0,9 & 1,0 & 1,1 & 0,8 & 1,5 \\
\hline & & & & & ENDEMICIDAD & 2,9 & 2,2 & 0,8 & 1,3 & 0,9 & 1,0 \\
\hline & & & & & RELICTISMO & 0,3 & 0,0 & 0,0 & 0,0 & 0,0 & 0,0 \\
\hline & & & & & CAR. FINÍCOLA & 1,3 & 1,1 & 0,6 & 0,7 & 0,6 & 0,0 \\
\hline & & & & & SUMA (INTER GLOBAL) & 6,4 & 4,3 & 2,2 & 3,1 & 2,3 & 2,5 \\
\hline & & & & & $\begin{array}{l}\text { F. GEOMORFOLÓGICA } \\
(\mathrm{x} 2)\end{array}$ & 17,4 & 19,1 & 16,5 & 14,0 & 14,6 & 14,0 \\
\hline & & & & & F. CLIMÁTICA & 9,3 & 9,3 & 9,1 & 7,2 & 7,8 & 7,0 \\
\hline & & & & ஹّ & F. HIDROLÓGICA & 9,1 & 9,4 & 8,3 & 7,7 & 8,0 & 8,0 \\
\hline & & & & & F. EDÁFICA & 8,0 & 8,0 & 8,0 & 7,2 & 7,2 & 7,0 \\
\hline & & & & & F. FAUNÍSTICA & 8,9 & 8,4 & 8,0 & 3,7 & 4,4 & 3,0 \\
\hline & & & & & SUMA (INMES GLOBAL) & 52,7 & 54,3 & 49,9 & 39,7 & 42,0 & 39,0 \\
\hline & & & SUMA & A (INNAT G & OBAL) & 91,4 & 88,7 & 81,3 & 64,8 & 64,7 & 56,5 \\
\hline & & & RIQUE & EST $(x$ 0'5) & & 6,1 & 6,2 & 5,2 & 6,3 & 4,3 & 4,5 \\
\hline & & & COBE & EST $\left(x 0^{\prime} 5\right)$ & & 4,7 & 5,2 & 4,1 & 4,8 & 4,0 & 3,5 \\
\hline & & & $\mathrm{FORH}$ & $\mathrm{AAB}$ & & 4,7 & 2,1 & 3,5 & 1,7 & 1,8 & 2,0 \\
\hline & & & FORE & ESP & & 3,4 & 9,9 & 15,5 & 7,3 & 8,2 & 1,0 \\
\hline & & SUI & MA (INN & NATFOR G & OBAL) & 110,4 & 112,1 & 109,6 & 84,8 & 83,0 & 67,5 \\
\hline & \multirow{6}{*}{\multicolumn{2}{|c|}{ INCUL }} & & \multirow{3}{*}{ FORETNO } & FORFIS & 2,6 & 2,0 & 2,0 & 1,0 & 1,0 & 1,0 \\
\hline & & & & & FORCUL & 2,0 & 1,7 & 1,9 & 1,5 & 1,0 & 1,0 \\
\hline & & & & & SUMA FORETNO & 4,6 & 3,7 & 3,9 & 2,5 & 2,0 & 2,0 \\
\hline & & & & \multicolumn{2}{|c|}{ VALOR PERCEPCIONAL (PER) } & 7,3 & 6,4 & 7,4 & 5,0 & 6,2 & 3,0 \\
\hline & & & & \multicolumn{2}{|c|}{\begin{tabular}{|l} 
VALOR DIDÁCTICO (DID) \\
\end{tabular}} & 7,6 & 6,4 & 5,3 & 4,7 & 3,8 & 1,0 \\
\hline & & & & \multicolumn{2}{|c|}{ SUMA (INCUL GLOBAL) } & 19,4 & 16,6 & 16,5 & 12,2 & 12,0 & 6,0 \\
\hline & \multicolumn{5}{|c|}{ SUMA (INCONTFOR GLOBAL) } & 129,9 & 128,7 & 126,1 & 97,0 & 95,0 & 73,5 \\
\hline & \multirow{5}{*}{\multicolumn{3}{|c|}{$\begin{array}{l}\text { PRIORIDAD DE } \\
\text { CONSERVACIÓN } \\
\text { (PRICON) }\end{array}$}} & PRES & IÓN DEMOGRÁFICA & 1,0 & 1,0 & 1,0 & 1,0 & 1,0 & 1,0 \\
\hline & & & & $\begin{array}{l}\text { ACCE } \\
\text { TRAI }\end{array}$ & $\begin{array}{l}\text { SIBILIDAD- } \\
\text { ISITABILIDAD }\end{array}$ & 4,3 & 4,0 & 5,1 & 5,3 & 5,4 & 5,0 \\
\hline & & & & AMEI & VAZAS ALTERNATIVAS & 3,9 & 2,4 & 1,3 & 2,7 & 1,4 & 1,0 \\
\hline & & & & $\begin{array}{l}\text { FACT } \\
\text { AMEI }\end{array}$ & $\begin{array}{l}\text { OR GLOBAL DE } \\
\text { JAZA }\end{array}$ & 9,1 & 7,4 & $\overline{7,4}$ & 9,0 & 7,8 & 7,0 \\
\hline & & & & PRIC & $\overline{\mathrm{ON}}$ & 1184,7 & 962,9 & 931,6 & 872,3 & 742,5 & 514,5 \\
\hline
\end{tabular}

Los resultados muestran una clara jerarquía. En primer lugar y, tal y como podía preverse, los paisajes forestales autóctonos muestran valoraciones generales superiores a aquellos bosques de repoblación con exóticas.

Haciendo un análisis más pormenorizado, se puede observar que, en general, los criterios que sumados dan lugar al valor territorial; rareza, endemicidad, relictismo y carácter finícola dan lugar a valores muy bajos, comparables a los obtenidos en latitudes muy superiores y bosques de tipo boreal (Finlandia y Noruega) (todavía no pu- 
blicados) y, por tanto, se alejan de los obtenidos por formaciones relativamente cercanas en otros ámbitos de carácter atlántico y mucho más con los obtenidos en ámbitos mediterráneos. Muy alejados quedan las formaciones de Chile mediterráneo donde este grupo de criterios daban valores muy altos (Lozano et al., 2015). Por lo tanto, existe una clara deficiencia en especies englobadas dentro de estos criterios. No obstante, los parámetros relacionado con los valores fitocenóticos y aquellos que se relacionan con los mesológicos son muy similares a los registrados en estos ámbitos atlánticos. En consonancia y, como consecuencia de los bajos valores territoriales, el INNAT muestra unos registros bastante discretos. No obstante, comparando las diferentes unidades que ahora nos ocupan, lo cierto es que ya deja entreverse una jerarquía u orden prelatorio claro que sigue el mismo que el descrito para los valores finales.

El segundo gran grupo de criterios engloba aquellos relacionados con la valoración complementaria para los paisajes forestales y que depende del número de taxones por estrato y unidad, la cobertura general de cada estrato por unidad, el número de microambientes existentes en cada una, así como la extensión de cada paisaje forestal. De esta manera, el orden jerárquico, entre los dos grandes grupos de paisajes forestales, sigue manteniéndose aunque existe un cambio en el orden prelatorio valorativo. El robledal aparece como el paisaje más valorado, seguido por el hayedo y el bosque de ribera. Esto se debe a la escasa extensión del bosque de ribera, de manera que el FORESP (prioiza las mayores extensiones) otorga mejores valores al hayedo y al robledal, por este orden. Este es, precisamente, el mismo orden que el registrado en cuanto a la superficie ocupada por cada una de las formaciones estudiadas. El pinar y los robledales rojos americanos, por su parte, aunque cuentan con extensiones importantes, se ven lastrados por las bajas valoraciones anteriores. No obstante, es curioso que el pinar cuente con el mayor valor para el RIQUEST, es decir, muestra el mayor número de especies por estratos de todos los paisajes estudiados. Ello se debe, claramente a que los pinares muestran una estructura muy abierta, con árboles relativamente separados, donde muchos fustes son muy longevos y se encuentran bastante afectados por enfermedades y, por tanto, las especies autóctonas, entran a formar parte también del complejo de esta formación.

En lo que respecta a los valores culturales, el orden prelatorio sigue siendo el mismo. Los bosques de ribera recogerían los mayores valores seguidos, por este orden, por los robledales, hayedos, pinares, robledales rojos americanos y, por último, los abetalesfalsos cupresales. La suma de los valores culturales más los naturales y los complementarios a las formaciones forestales dan un orden prelatorio igual, donde las tres formaciones autóctonas siguen aventajando en bastantes puntos a los bosques de repoblación con especies alóctonas. En cualquier caso, la diferencia entre las tres formaciones del primer grupo es de 3,8 puntos, muy baja. Mientras, las diferencias entre las formaciones del segundo grupo son de 23,5 puntos, aunque entre el pinar y el robledal rojo americano existe una exigua diferencia de 2 puntos.

En lo que respecta al factor global de amenaza, las diferencias más notables se obtienen dentro del capítulo de amenazas alternativas. Al respecto, el valor más alto se 
registra para el bosque de ribera y se deriva de la posible existencia de riadas que pueden dar lugar a pérdidas de ciertas porciones o sectores de esa formación. También otros riegos derivados de actuaciones antrópicas han generado impactos importantes en el pasado y en la actualidad, por una parte se trata de la red de caminos que, en gran medida, por lo menos los más importantes, discurren en paralelo a los cauces fluviales invadiendo el dominio del bosque de ribera. Por otra parte, tradicionalmente han existido intervenciones sobre los cauces (canales de deriva, ferrerías, molinos, aceñas, que pueden modificar el caudal o trazado del río y, por tanto, poner en riesgo ciertos sectores dominados por este bosque ripario.

También la puntuación registrada por el pinar es relativamente alta y se debe a la existencia de mortandades bastante elevadas entre los pinos. Existe una gran proporción de fustes muertos, lo que hace suponer que, o bien por edad, o por otro motivo (hongos, enfermedades, otro tipo de plagas) el riesgo de que estas masas se vayan perdiendo y sean sustituidas por la dinámica general es cierto. Se podría pensar que esta dinámica sería la adecuada al ser el propio pinar una formación alóctona pero lo cierto es que cierto número de hectáreas de esta formación añade mayor biodiversidad y una mayor heterogeneidad paisajística lo cual debe ser valorado positivamente.

\section{Conclusiones}

De un total de 35 inventarios biogeográficos realizados se han derivado 6 sininventarios, que muestran paisajes forestales bien diferenciados y constituyen una buena representación de la vegetación forestal-frutescente del sector pirenaico occidental. En concreto, se ha registrado un total de 87 especies vegetales en menos del 5\% total del territorio objeto de estudio. De ellas, 59 son herbáceas, 18 matas y trepadoras y tan sólo 10 árboles y arbustos.

Existe una clara diferencia entre los paisajes forestales de especies autóctonas de aquellos de repoblación con especies alóctonas. Dentro de los tres primeros existe una prelación clara liderada por el bosque de ribera, seguido del robledal y el hayedo. Por su parte, dentro del segundo grupo también se sigue un orden que no varía estando en primer lugar el pinar de pino silvestre, el robledal de roble rojo americano y, por último, el abetal-falso cupresal. Los del primer grupo cuentan con puntuaciones bastante superiores a los del segundo.

El paisaje que mayor valoración obtiene es el bosque de ribera con 1.184,7 puntos, seguido del robledal con 962,9 puntos, el hayedo con 931,6 puntos, el pinar con 872,3 puntos, el robledal rojo americano con 742,5 puntos y, por último y con unas valoraciones muy bajas equiparables a matorrales cuasi monoespecíficos de Ulex europaeus, el abetal-falso cupresal. 
Aun y todo las valoraciones son bastante modestas si tenemos en cuenta el resto de trabajos referenciados a lo largo del texto y la bibliografía. La cobertura extensa de bosques, las fuertes pendientes, los escasos y oligótrofos suelos y una cierta uniformidad del paisaje hacen que los valores naturales sean realmente bajos. Por otra parte, al tratarse de un espacio protegido y con una gestión de protección a ultranza hace que el factor global de amenaza sea ciertamente bajo y, por ende, sean bajas también las valoraciones finales. No obstante, son muy similares a paisajes forestales enclavados dentro del Colsacabra, en la Cordillera Transversal (Lozano et al, 2014), así como en el sector de la comarca de Bortziri, muy cercanos a los de Artikutza.

Finalmente, es interesante cotejar el valor global final (PRICON) más elevado obtenido en este sector de Artikutza (los 1.184,7 puntos del bosque de ribera) con los registrados por nuestro equipo de investigación en diversos ambientes forestales donde hemos tenido oportunidad de trabajar. En la Península Ibérica ninguna unidad analizada sobrepasa los 3000 puntos. Así, el robledal de Quercus pyrenaica llega a alcanzar los 1430, el quejigal de Quercus faginea 1380 y el encinar de Quercus rotundifolia 1365; siendo los valores más altos los del alcornocal atlántico de Quercus suber (2623,9; la puntuación más elevada en territorio europeo), tremolinar de Populus tremula $(2384,5)$ y robledal de Quercus robur $(2383,5)$ (Cadiñanos, Lozano y Quintanilla, 2011). El récord absoluto (superior a 3500 puntos) registrado hasta el momento por nuestro equipo corresponde al bosque con palma (Jubaea chilensis) de la región chilena de Valparaíso (Lozano et al., 2015). Por todo ello se colige algo que ya fue apuntado anteriormente, las bajas puntuaciones generales de los paisajes forestales de Artikutza.

\section{Agradecimientos}

Este artículo ha sido posible gracias al proyecto de investigación: "Valoración paisajística, ambiental y socioeconómica de los espacios agrarios de alto valor natural de la CAE. (GIU10/07)".

\section{Bibliografía}

Adamds, M.D., Law, B.S., y French, K.O., 2009. Vegetation structure influences in the vertical stratification of open and edge-space aerial foraging bats in harvested forests. Forest Ecology and Management, 258, pp.

Aizpuru, I., Areta, P., Barrio, L.D., Catalán, P., Mendiola, I. eta Zorrakin, I., 1989. Guia ecologica de Artikutza. Naturaleza y buella bumana. Donostia-San Sebastian: Aranzadi Zientzia Elkartea, 103 pp.

Ausden, M., 2007. Habitat management for conservation: A handbook of technicques. New York: Oxford University Press. 
Balling, J.D., Falk, J.H., 1982. Development of visual preference for natural environments, Environ. Behav 14, 5-28.

Blaschke, T., 2005. The role of thespatial dimension within the framework of sustainable landscapes and natural capital, Landsc. Urban Plan 75, 198-226.

Bourassa, S.C., 1990. A paradigm for landscape aesthetics, Environ. Behav., 22, 787-812.

Cáncer Pomar, L.A., 1999. La degradación y la protección del paisaje, Geografía menor. Madrid: Cátedra, $247 \mathrm{pp}$.

Cadiñanos, J.A. y Meaza, G., 1998a. Bases para una Biogeografia aplicada. Criterios y sistemas de valoración de la vegetación. Logroño: Geoforma ediciones, 144 pp.

Cadiñanos, J.A. y Meaza, G., 1998b. Nueva propuesta metodológica de valoración del interés y de la prioridad de conservación de la vegetación, Mauleon, Actas del Colloque International de Botanique Pyreneo-Cantabrique.

Cadiñanos, J.A. y Meaza, G., 2000. Metodología complementaria de evaluación de ecosistemas forestales. Inédito.

Cadiñanos, J.A., Meaza y Lozano, P.J. 2002. Valoración del interés y de la prioridad de conservación de bosques y comunidades preforestales de Larra (Alto Pirineo Navarro), La Gomera, La Biogeografia: ciencia geográfica y ciencia biológica. Actas del II Congreso Español de Biogeografía.

Cadiñanos, J.A., Diaz, E., Ibisate, A., Lozano, P., Meaza, G., Peralta, J., Ollero, A. y Hormaetxea, O., 2002. Aplicación de una metodología de valoración de la vegetación a riberas fluviales: ensayo en el río Butrón (Bizkaia), Zaragoza, Aportaciones geográficas en memoria del Prof. L. Miguel Yetano Ruiz, 65-88.

Cadiñanos, J.A., Lozano, P.J. y Quintanilla, V., 2011. Propuesta de marco integrado para la valoración biogeográfica de los espacios Red Natura 2000 de la Comunidad Autónoma del País Vasco. El ejemplo de Gárate-Santa Bárbara (Guipuzcoa), Madrid: Boletín de la Asociación de Geógrafos Españoles 57, 33-56.

Cadiñanos, J.A., Lozano, P. y Quintanilla, V., 2011. Propuesta de marco metodológico integrado para la valoración biogeográfica de espacios Red Natura 2000 de la comunidad autónoma del País Vasco. El ejemplo de Gárate-Santa Bárbara (Guipúzcoa). Madrid, Boletín de la AGE 57, 33-56.

Comunidades Europeas., 2000. Gestión de espacios Natura 2000. Disposiciones del artículo 6 de la Directiva 92/32/CEE sobre hábitats. Luxemburgo: Oficina de Publicaciones Oficiales de las Comunidades Europeas.

Daily, G.C., Söderquvist, S.A., Arrow, K., Drasgupta, P., Ehrlich, P.R., Folke, C., Jansson, A., Jansson B., Kautsky, N., Levin, S., Lubchenco, J., Mäler, K., Simpson, D., Starrett, D., Tilman, D., Walker, B., 2000. Ecology: The Value of Nature and the Nature of Value. Science 289, 395-401.

De Groot, R.S., Wilson, M.A., Boumans, R.M.J., 2002. A typology for the classification, description and valuation of ecosystem functions, goods and services, Ecol. Econ. 41, 393-408.

De la Barrera, F., Reyes-Paecke, S. y Meza, L., 2011. Análisis del paisaje para la evaluación ecológica rápida de alternativas de relocalización de una ciudad devastada. Revista Chilena de Historia Natural 84, 181-194. 
Drever, M. y Martin, K., 2010. Response of woodpeckers to changes in forest health and harvest: Implications for conservation of avian diversity. Forest Ecology and Management 259, 958-966.

Ekilan S.L., 2012. Proyecto de Ordenación forestal y Plan de Gestión del LIC de Artikutza. Ansoain: Ayuntamiento de Donostia y Gobierno de Navarra, 120 pp.

Faith, D.P. \& Walker, P.A., 1996. Integrating conservation and development: effective trade-offs between biodiversity and cost in the selection of protected areas. Biodiversity and Conservation 5, 431-446.

Fisher, B., Turner, R.K., Morling, P., 2009. Defining and classifying ecosystem services for decision making. Ecol. Econ. 68(3), 643-653.

Forman, R., 1995. Land mosaics. The ecology of landscapes and regions. Cambridge UK, Cambridge University Press.

González-Bernáldez, F., Parra, F., 1979. Dimensions of landscape preferences from pairwise comparisons. In: Elsner, G.H., Smardon, R.D. (Eds.), Our National Landscape. General Technical Report PSW-35. USDA Forest Service, Berkeley, CA, USA.

Grez, A., Simonetti, J. y Bustamante, R., (eds) 2006. Biodiversidad en ambientes fragmentados de Chile: Patrones y procesos a diferentes escalas. Santiago de Chile, Editorial Universitaria.

Hagar, J., 2007. Wildlife species associated with non-coniferous vegetation in Pacific Northwest conifer forests: A review. Forest Ecology and Management 246, 108-122.

Hervé, D., 2010. Noción y elementos de justicia ambiental: Directrices para su aplicación en la planificación territorial y en la evaluación ambiental estratégica. Valdivia, Revista de Derecho 23, 9-36.

Kaltenborn, B.P., Bjerke, T., 2002. Associations between environmental value orientations and landscape preferences. Landscape and Urban Planning 59, 1-11.

Kaplan, R., Kaplan, S., 1989. The Experience of Nature. Cambridge UK, Cambridge Univerisity Press.

Leopold, L.B., 1969. Quantitative comparison of some aesthetic factors among rivers. U.S. Geological Survey 620, Washington, D.C., U.S.A Department of the Interior, 1-16.

Litton, R.B., 1972. Aesthetic dimensions of the Landscape. Natural Environments Studies in Theoretical an Applied Analysis, Baltimore. Ed. John V. Krutilla, The John Hopkins University Press, 262-291.

Lozano, P.J., 2006. Régimen precipitacional en el norte de Navarra y Guipúzcoa. ¿Record peninsular y europeo?. Murcia, Nimbus 17-18, 125-144.

Lozano, P.J., Cadiñanos, J.A., Longares, L.A., Cid, M.A., 2007. Valoración Biogeográfica de los tipos de bosque en la combe de Huidobro (Parque Natural de las Hoces del Ebro-Burgos). Ávila, Actas del $4^{\circ}$ Congreso Español de Biogeografía 19.

Lozano, P.J. y Cadiñanos, J.A., 2009. Propuesta de marco metodológico integrado para la valoración de Espacios de la Red Natura 2000 de la Comunidad Autónoma del País Vasco. El ejemplo de Gárate-Santa Bárbara (País Vasco). Malaga, Biogeografía Scientia Biodiversitatis 199-206.

Lozano P.J., Cadiñanos,J. A., Latasa, I. y Meaza, G., 2013. Caracterización y valoración biogeográfica de los pinares de Pinus uncinata del karst de Larra (Alto Pirineo Navarro) para su ordenación y gestión. Zaragoza, Geographicalia 63-64, 95-120. 
Lozano P.J., Cadiñanos, J.A., Latasa, I., Quintanilla, V., Meaza, G., 2015. Caracterización, valoración y evaluación de los paisajes vegetales de Chile Mediterráneo. Madrid, Boletín de la Asociación de Geógrafos Españoles 67, 14-32.

Lyons, E., 1983. Demographic correlates of landscape preference. Environ. Behav., 15, 487-511.

Mc Neill J.R., 2000. Algo nuevo bajo el sol. Historia medioambiental del mundo en el siglo XX. Madrid, Alianza ensayo, 504 pp.

Meaza, G., Cadiñanos, J.A., Lozano, P.J., 2006. Valoración biogeográfica de los bosques de la reserva de la biosfera de Urdaibai (Vizcaya). Urdaibai, Actas del III Congreso Español de Biogeografía 399-411.

Rivas-Martínez, S., 2007. Mapa de series, geoseries y geopermaseries de vegetación de España. Memoria del mapa de vegetación potencial de España. Madrid, Itinera Geobotanica 17, 5-436.

Sagastibeltza, E., Lozano P.J., Herrero, X., 2014. Nafarroako Bortzirietako baso-landaredien paisaien inbentariazioa, karakterizazioa eta balorazio biogeografikoa. Donostia-San Sebastian, $L u-$ rralde $37,97-133$.

Strijker, D., Sijtsma, F.J. y Wiersma, D., 2000. Evaluation of nature conservation: An application to the Dutch Ecological Network. Environmental and Resource Economics 16, 363-378. 
